\title{
Determinants of Eye Lid Surgical Care Utilization Among Trachomatous Trichiasis Patients in Rural Communities: In the Case of Basoliben District, North West Ethiopia
}

\author{
Kassahun Ketema Aredo, ", Adugna Birhanu Kebede ${ }^{2}$, Mekonin Aychiluhim ${ }^{3}$, \\ Mulatu Ayana Hordofa ${ }^{1}$ \\ ${ }^{1}$ Department of Public Health, College of Medicine and Health Sciences, Debre Markos University, PO Box: 269, Debre Markos, Ethiopia \\ ${ }^{2}$ Debre Markos, East Gojjam Zone Health Office, Carter Center Trachoma Control Project Coordinator, PO Box: 17, Debre Markos, Ethiopia \\ ${ }^{3}$ Department of Medicine, GAMBY Collage of Medical Sciences, Bahar Dar, Ethiopia
}

\author{
Email address: \\ kasyon33@Gmail.com (K. K. Aredo), Adugna@yahoo.com (A. B. Kebede), mekachy@yahoo.com (M. Aychiluhim), \\ mulatuayana@gmail.com (M. A. Hordofa)
}

\section{To cite this article:}

Kassahun Ketema Aredo, Adugna Birhanu Kebede, Mekonin Aychiluhim, Mulatu Ayana Hordofa. Determinants of Eye Lid Surgical Care Utilization among Trachomatous Trichiasis Patients in Rural Communities: In the Case of Basoliben District, North West Ethiopia. American Journal of Internal Medicine. Vol. 2, No. 6, 2014, pp. 156-161. doi: 10.11648/j.ajim.20140206.20

\begin{abstract}
Background: Trachomatous trichiasis is worldwide leading cause of blindness. Surgical intervention is one of the means of prevention of this blindness. Objectives: To assess determinants of eyelid surgical care utilization among trichiasis patients and associated factors. Method: A community based cross- sectional study design was employed. Sample size was determined using a single population proportion formula. Result: Among 348 interviewed patients, 253(72.7\%) were in the age group of 16-45 years. Two hundred eighty nine (83.1\%) were non-operated and 59(16.9\%) operated trichiasis cases. Bilateral eye trichiasis cases were $245(70.4 \%)$ and $195(56 \%)$ of them were ill for 5-8 years. Ninety-seven $(27.9 \%)$ believe treatment of trichiasis is surgery. Reasons for not using trichiasis surgery were distance of treatment center $\left(\mathrm{X}^{2}=36.2\right)$, lack of awareness $\left(X^{2}=46.8\right)$, and false beliefs about treatment $\left(X^{2}=14.6\right)$. Conclusion: Associated factors were distance, lack of information, lack of time, fear of surgery.
\end{abstract}

Keywords: Trachomatous Trichiasis, Surgical, Determinants, Community, Ethiopia

\section{Introduction}

Trachoma is a preventable and treatable disease caused by Chlamydia trachomatis. It is the most common infectious cause of blindness worldwide. Trachoma affected 146 million active cases, 10.6 million with trichiasis and 5.9 million blind $[1,2]$. Trachomatous trichiasis (TT) is one of the stages of trachoma in which one or more eyelashes from an in turned eyelid rub on a cornea [3]. If trichiasis is not treated surgically, it may eventually lead to corneal opacity and blindness [4]. The discomfort from trichiasis results in an economic burden comparable to visual impairment caused by trachoma [5].Trachoma is the second most common cause of blindness in Ethiopia [6] where trichiasis affects 3\% of people above 14 years of age [6].The World Health Organization has launched an initiative to eliminate blinding trachoma by the year 2020 using the SAFE strategy: Surgery,
Antibiotic, Facial Cleanliness and Environmental improvement [7]. Surgical correction of the upper eyelid using tarsal rotation procedure is the most effective intervention for trichiasis [8]. Trachomatous trichiasis surgery treatment is provided free or subsidized in most trachoma endemic settings. However, only $18-66 \%$ of TT patients attend for surgery [9]. Despite the scale-up of surgical services in recent years, current surgical activity is not effectively tackling the backlog. There are multiple provider- and patient-specific barriers. Moreover, there were challenges for retention of TT surgeon for provision of service [10].

Low uptake of TT surgery has always been of concern for the success of the 'S' (surgery) trachoma-control strategy [11]. Unfortunately, surgical coverage in affected communities in 
Africa is lower than 50\% [12]. In Ethiopia, the overall coverage of trichiasis surgery was $41 \%$ [13]. In trachoma endemic areas of central Ethiopia utilization of eye care services in general was $27.8 \%$ [14].

The national blindness and trachoma program were started in 1976. Vision 2020 was launched in 2002 in Ethiopia and the federal ministry of health has identified 2020 as the target for eliminating blinding trachoma as public health problems in Ethiopia, although the Amhara region plans to eliminate blinding trachoma by 2015 [15].

In 2005 and 2006, the federal ministry of health of Ethiopia conducted a national blindness and low vision survey in collaboration with international partners who were deployed in the prevention of blindness. The finding of the survey showed that the prevalence of blindness in Ethiopia at $1.6 \%$ is among the highest in the world $[15,16]$. Blindness from trachoma was attributed to $12 \%$ of all blindness nationally which is second to cataract. Trachomatous trichiasis among adults 15 years and older was 3.1\% [16].

Surgery in Ethiopia Trachomatous trichiasis is offered free of charge through both approaches: routine health service provision and surgical outreach campaigns [16,17]. Of 832 districts in Ethiopia, 593 are priority districts for surgery. Approximately one-third of these districts have active surgeons. At the end of 2011, the federal ministry of health estimated that $1,053,813$ TT cases need to be operated in order to clear the backlog [17]. The federal ministry of health has conducted 66,409 surgeries. Since 2001; the national program has achieved a total of 442,868 TT surgeries throughout the country. The national program estimates that there remains a back log of approximately one-million non operated trichiasis cases [6].

Integrated eye care workers were trained to perform the surgery and they were certified using the World Health Organization's guide lines upon successful completion of trainings. TT surgeons provided all the necessary materials that is consumable and non consumables which helps to conduct TT surgery either during static service or during the campaign time. In Ethiopia antibiotics distribution would takes place by using the existing health infrastructures, which is through villages' health extension workers, community health volunteers, field supervisors all are implementers responsible to address all community members to take the antibiotics during the distribution campaign [6]. During surgery and antibiotics distribution campaigns health extension workers play a key role in social mobilization to advocate the benefits of treatment with those at risk of trachoma infection or blindness from trichiasis [18,19].

The aim of this study was to identify determinants of eyelid surgical treatment utilization and associated factors among trichiasis patients

\section{Methods and Materials}

\subsection{Study Design and Area}

A community based cross-sectional study was employed in
Baso Liben district, East Gojjam Zone of Amhara Regional state, Ethiopia in September, 2013. The area has two agroclimatic divisions: $54 \%$ low altitude and $46 \%$ medium altitude with an altitude of less than 1500 meters and 15002500 meters above sea level, respectively. According to the Central Statistical Agency in 2007, Baso Liben has total population of 137,470 , of whom $67,594(49.2 \%)$ are men at website [20].

The district constitutes 25 rural villages of which five villages were included in the study. In the district there are five health centers and twenty five health posts. The total backlogs were 5,417 of whom 2,322 had received the surgery service and the remaining backlogs that didn't get the service were 3,095.

The target population was all previously operated and nonoperated TT patients living in the district. The study population was all trichiasis patients living in the randomly selected five villages. The study included operated and nonoperated TT patients whose ages were greater than 15 years where as severely ill and who had operation before two years or more were excluded.

\subsection{Sampling Methods}

The sample size was calculated using single population proportion formula by taking into consideration $7.1 \%$ prevalence rate of TT from unpublished baseline survey result of Amhara National Regional State health bureau conducted in 2008. Trained health extension workers in each village were deployed in tracing for surgical and antibiotic distribution campaign in 2012 records of which 769 had traced and enrolled in the district. A multistage stratified sampling technique proportional to size was applied. Five from 25 villages were randomly selected by using the lottery method. Three hundred forty eight TT cases were interviewed in the selected villages of which 289 were unoperated and 59 operated.

\subsection{Operational Definition}

- Epilation: is the act of removing in turned eyelashes mechanically from the eyes by local device

- Trichiasis: in turning of eyelashes to touch the eye secondary to trachoma

- Trained integrated eye care workers: Are successfully completed the basic training at secondary eye unit where the ophthalmologists are avail and has to be certified as per the World Health Organization certification criteria to be legally practice

- Trachomatous trichiasis: if the cases at present at least one eye lash rubbing the eye ball or Epilating recently or If the clientis having a device for epilation to say that patient is having trichiasis.

\subsection{Data Collection Procedure}

A semi-structured questionnaire was used for data collection. One day training was given for five data collectors and two supervisors. The data collectors were integrated eye 
care workers who were currently deployed and working to their respective health centers. The two supervisors were recruited from health office of Basoliben district. A supervision system was established and practical throughout the data collection period and pre-test of the questionnaire carried out in one of the village adjacent to the study district. The collected data were checked daily for consistency, completeness, clarity and accuracy throughout the data collection process.

\subsection{Data Processing and Analysis}

Data were entered using epi data statistical software version 3.1 and analyzed using SPSS version 20. Descriptive statistics using tables and graphs were presented. In an attempt to identify the association of explanatory variables on the outcome variable was seen, chi-squire analyses was applied.

\subsection{Ethical Considerations}

The study was approved by ethical review committee of the Debre Markos University, College of Medicine and Health Sciences. Support letter and permission was obtained from the district health office and village administrators. The purpose of the study was explained to all eligible respondents and verbal consents were obtained and recorded on consent form. Written consent was not taken because of the high rate of illiteracy among respondents, thus approved by the committee. Study subjects were appointed for treatment for this free of charge.

\section{Result}

\subsection{Socio-Demographic Characteristics}

Three hundred forty eight respondents were selected for the study with a $100 \%$ response rate. The most affected $253(72.7 \%)$ age was between 45-61 years and two hundred seventeen $(62.4 \%)$ were female. Two hundred eighty nine (83.1\%) study participants were non-operated and 59(16.9\%) previously operated trichiasis patients. Women were $217(62.4 \%)$ while $131(37.6 \%)$ were men. The majority $292(83.9 \%)$, trichiasis cases could not read and write. One hundred fifty seven $(45.1 \%)$ of respondents' monthly income was less than 1000 Ethiopian Birr (Table 1).

\subsection{Determinant Factors for Utilization of Trichiasis Surgery}

Two hundred forty five (70.4\%) of respondents had trichiasis on both eyes and 234(67.2\%) of them were having eye illness for more than five years. Among study subjects $253(72.7 \%)$ were practicing epilation for more than one year. Two hundred eighty two (81\%) did not know any person who received trichiasis surgery. Some of them did not hear about surgery complication and its outcome.

Regarding clinical complains, 289(83\%) of them had reported eyelash in-turn was causing problems manifested by persistent pain $227(78.5 \%)$, occasional pain 50(17.3\%) and self reported visual impairment 12(4.2\%). From respondents $52(14.9 \%)$ responded that trichiasis is caused by trachoma the rest respondents were having misconceptions. They believed that presence of smoke for house hold tasks $111(31.9 \%)$, tear due to crying $86(24.7 \%)$, due to dust or dirt $76(21.8 \%)$, hereditary $10(2.9 \%)$, hard work $6(1.7 \%)$, naturally growing eyelashes in ward $5(1.4 \%)$ were stated to as causes of trichiasis.

Table 1. Socio demographic characteristics of study participants in Basoliben district, north West Ethiopia, December 2013.

\begin{tabular}{llll}
\hline Variables & Category & Frequency & $\begin{array}{l}\text { Percentage } \\
(\%)\end{array}$ \\
Age in years & $16-45$ & 253 & 72.7 \\
& $46-60$ & 95 & 26.3 \\
Sex & Male & 131 & 37.6 \\
& Female & 217 & 62.4 \\
& Married & 259 & 74.4 \\
Marital status & Single & 35 & 10.1 \\
& Separated & 19 & 5.5 \\
Religion & Widowed & 35 & 10.1 \\
Educational & Orthodox Christian & 348 & 100 \\
status & Cannot read and write & 292 & 83.9 \\
Occupation & Can to read \& write & 56 & 16.1 \\
& Farmer & 345 & 99.1 \\
Average & day labourer & 3 & 0.9 \\
monthly & $<1000$ & 157 & 45.1 \\
income & $1000-1999$ & 138 & 39.7 \\
& 2000-2999 & 43 & 12.4 \\
\hline
\end{tabular}

Table 2. Factors determining Trachomatous Trichiasis surgery utilization in Basoliben District, North West Ethiopia, December, 2013.

\begin{tabular}{llll}
\hline Variables & Category & Frequency & $\begin{array}{l}\text { Percent } \\
\text { (\%) }\end{array}$ \\
\hline \multirow{3}{*}{ Affected eyes } & Left & 63 & 18.1 \\
& Right & 40 & 11.5 \\
Duration of illness in & Both & 245 & 70.4 \\
year & $<5$ years & 114 & 32.8 \\
History of epilation & Yes years & 234 & 67.2 \\
Time of epilation & No & 253 & 72.7 \\
practice & $\geq 1$ year & 95 & 27.3 \\
Knowing about & Yes & 105 & 30.2 \\
trichiasis surgery & No & 243 & 69.8 \\
Problem after & Yes & 66 & 19 \\
trichiasis surgery & No & 282 & 81 \\
Outcome of trichiasis & No improvement & 4 & 23.7 \\
surgery & Recurrence & 8 & 76.3 \\
Knowing where & Worsening of vision & 2 & 28.6 \\
trichiasis surgery s & Yes & 129 & 57.1 \\
Walking distance HI & No & 219 & 14.3 \\
& $>2$ hours & 133 & 37.1 \\
\hline & $>2$ hours & 215 & 62.9 \\
\hline
\end{tabular}

Among respondents' previously, operated trichiasis cases were nearer to the health facility than the non-operated one. Two hundred fifteen (61.8\%) trichiasis cases were living more than two hours walk away from health center (chisquare $=36.2, \mathrm{df}=1, \mathrm{p}<0.001)$. Regarding institution where operation took place, $42(71.2 \%)$ were in the nearby health centers, $12(20.3 \%)$ in the health post and $5(8.5 \%)$ in nearby 
referral government hospital. From the total 59 operated trichiasis, $45(76.3 \%)$ reported that the outcome of the procedure had been good. Most of respondents 219(62.9) did not know any institution where trichiasis surgery was given (chi-square $=46.8, \mathrm{df}=1, \mathrm{p}<0.001)$ (Table 2).

Some of the respondents $97(27.9 \%)$ believed that treatment of trichiasis was surgery; the remaining of them had false beliefs (chi-square $=14.6, \mathrm{df}=3, \mathrm{p}<0.01$ ). One hundred two (43.7\%) believed epilation with local device, application of eye drop or eye ointments topically $22(6.3 \%)$, face washing with soap and water, herbal medication and there were those who did not know 21(6\%) (Figure 1).

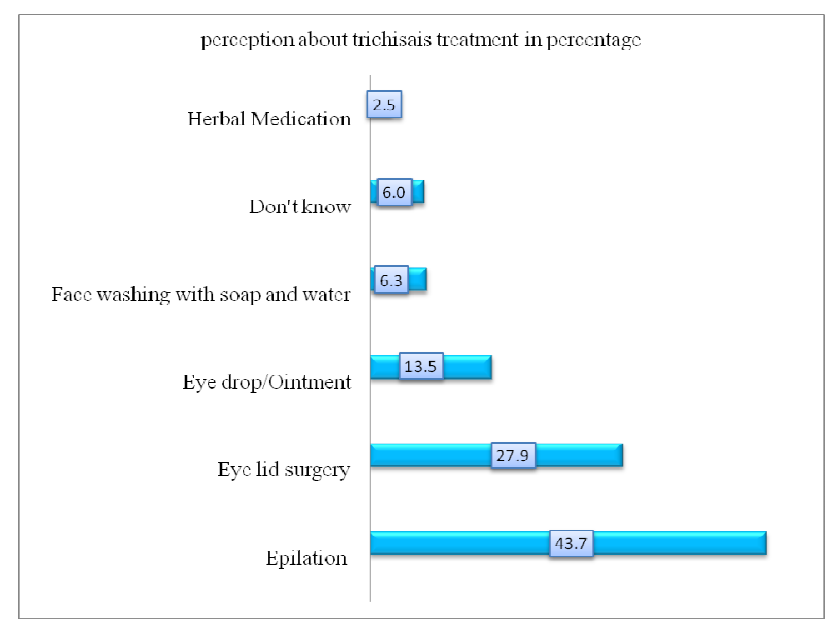

Figure 1. Perception of trichiasis treatment of study subjects in Basoliben district, 2013

The major reasons for not using trichiasis surgery were being busy on household tasks 124 (35\%), fear of surgery $111(32 \%)$, lack of money for transport, food and lodging cost $69(20 \%)$ given by the respondents (chi-square $=151.4$, $\mathrm{df}=5, \mathrm{p}<0.001$ ) (Figure 2). Being busy 81(69.2) with household tasks and lack of transport, food cost and lodging $36(55.4 \%)$ were challenges for seeking surgical treatment of more women than men.

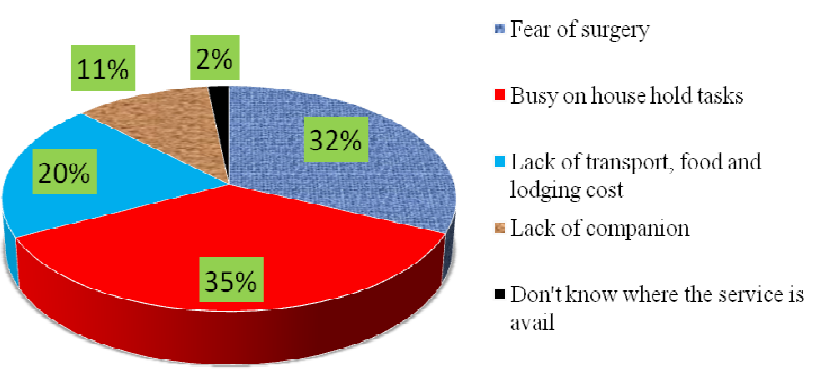

Figure 2. Main reasons of refusal not having trichiasis surgery service in Basoliben district, December, 2013.

\section{Discussion}

The prevalence of active trachoma is decreasing, due to the combined impact of mass antibiotic treatment campaigns, environmental improvements and socio-economic development in endemic regions. Trachomatous trichiasis remains a major cause of blindness worldwide; even with the expansion of trachoma-control program is in progress. There is less evidence for a decline in the number of people with TT $[1,12]$. Trachomatous trichiasis surgery has provided free or sub-sidized in most trachoma endemic settings like Ethiopia. However, only $18-66 \%$ of TT patients attend for surgery [9]. Here we were interested to investigate the determinant factors of uptake of surgery in the area.

Even though, it is insignificant, trichiasis affected 16-45 more than 45 years old $(72.7 \%$ versus $26.3 \%)$ people did. This finding contradicts with the in seventeen-outreach campaigns in Amhara Region [9]. The most possible reason for these could be difference in sample size, altitude (most of our respondents were from low land) and awareness status of the community.

All cases who were interviewed had shown manifestations of trichiasis and most of them had developed bilateral trichiasis $(70.4 \%)$ for more than five years $234(67.2 \%)$, thus indicates that blindness is indispensable unless appropriate surgical intervention is provided rapidly. Despite presence of free of charge trichiasis surgical treatment most of study subjects were using other means of treatment like epilation. This could be due their lack of information, inaccessibility and misconception about trichiasis surgery treatment.

In the study, women were more affected $(62.4 \%$ versus $37.6 \%$ ) than men. This result is in line with the study done in four areas across Ethiopia [13]. This may be due to women socio-economic status, educational and cultural issue. The study revealed that $292(83.9 \%)$ trichiasis cases could not read and write. This result is in consistent with the study in Tanzania and Ethiopia [12, 13]. It could be due to lack of information, less access to information and awareness. Seventy-two $(20.7 \%)$ of the study subjects had awareness of trichiasis surgical treatment. It inline with study in Tanzania [12] however, it is very low when compared with other study in Ethiopia [9]. This difference could due to difference in sample size, study subject, study area difference and time of the study.

In the study people who live more than two hours of walk $215(61.8 \%)$ from the nearby health institution providing surgery were less likely to have had eyelid surgery. This was consistent with the study done in North West Ethiopia and Tanzania $[10,12]$. This is possibly due to fear of long walk from the health institutions to the dwelling houses with eye dressing after the surgery has done and need of companion.

In this study, 251(72.1\%) had false beliefs about trichiasis treatment. This was in line with study in surveys conducted in Ethiopia between 2002 and 2008[13]. However, it was larger in this study $(72.1 \%$ versus $17 \%)$ than survey. This could be due to difference in sampling method and study population. Among those false beliefs epilation 102 (43.7\%) with local device was the leading one.

The study revealed that major reasons for not using trichiasis surgery by respondents were being busy on house hold tasks $124(35 \%)$, fear of surgery $111(32 \%)$, lack of money for transport, food and lodging cost $69(20 \%)$. Similar findings were reported in the study done in 17 surgical 
outreach campaigns in Amhara Region of Ethiopia [9]. However, the findings were lower than this study. This could be due to difference in sample size, data collection time and more infrastructures.

A limitation of this study is that the study included selfreported cases, which possibly had missed asymptomatic ones. Hence, the possible information was collected from trichiasis cases who voluntarily attended the interview.

\section{Conclusion}

In this community, many people were affected by Trachomatous trichiasis. Most affected once were women and middle age (31-45) group. Major associated factors were distance from health institution, lack of knowledge about where to get service, lack of time, fear of surgery, indirect cost and misconception about trichiasis treatment.

Extending of trichiasis surgery at peripheral health centers and screening program which identify and facilitate transport to health facilities for trichiasis patients is one approach. The indirect burden of accessing eye care on the family may discourage patients to have surgery earlier and by improving the efficiency of existing services. Health education and promotion should give emphasis to improve misconception about treatment and cause of trichiasis. Promotion of services must be gender sensitive, ensuring that specific characteristics of the socio cultural roles of women be considered in order to improve uptake among women. Further investigation may enable identification of the more determinant factors to uptake of surgery.

\section{Acknowledgements}

We acknowledge The Carter Center Ethiopia (TCCE) and Amhara National Regional State malaria and trachoma control project coordination office for transportation and provision of necessary documents. Our special appreciation deserves to Dr Zerihun Tadesse the Carter Center Ethiopia country representative for his significant role in allowing by investing time to attend the training session without his willingness or sincerity it would have been impossible to materialize the desired achievements.

The support obtained from Amhara Regional Health Bureau, East Gojjam Zone Health and data collectors and all the study participants were highly acknowledged. We are deeply indebted to Dr Alemu Disassa who was editing of the manuscript.

\section{References}

[1] Mariotti SP, Pascolini D, Rose-Nussbaumer J. Trachoma (2009) Global magnitude of a preventable cause of blindness. Br J Ophthalmol 93:563-568.

[2] Trachoma: A Blinding Scourge from the Bronze Age to the Twenty First Century, Taylor, HR,Haddington Press, Victoria Australia, 2008.
[3] Thylefors B, Dawson CR, Jones BR, West SK, Taylor HR (1987) A simple system for the assessment of trachoma and its complications. Bull World Health Organ 65: 477-483.

[4] Resnikoff S, Pascolini D, Etya'ale D, Kocur I, Pararajasegaram R, et al. (2004) Global data on visual impairment in the year 2002. Bull World Health Organ 82: 844-851.

[5] Frick KD, Keuffel EL, Bowman RJ (2001) Epidemiological, demographic, and economic analyses: measurement of the value of trichiasis surgery in The Gambia. Ophthalmic Epidemiol 8: 191-201.

[6] Berhane Y, Worku A, Bejiga A, Adamu L, Alemayehu W, et al. (2007) Prevalence and causes of blindness and Low Vision in Ethiopia. Ethiop J Health dev. 21(3): 204-210.

[7] World Health Organization (2006) Trachoma control: a guide for program managers.

[8] Yorston D, Mabey D, Hatt S, Burton M (2006) Interventions for trachoma trichiasis. Cochrane Database Syst Rev: CD004008.

[9] Rajak SN, Habtamu E, Weiss HA, Bedri A, Zerihun M, et al. (2012) Why Do People Not Attend for Treatment for Trachomatous Trichiasis in Ethiopia? A Study of Barriers to Surgery. PLoS Negl Trop Dis 6(8): e1766.

[10] Habtamu E, Rajak SN, Gebre T, Zerihun M, Genet A, et al. (2011) Clearing the backlog : trichiasis surgeon retention and productivity in Northern Ethiopia. PLoS Negl Trop Dis 5: e1014.

[11] West ES, Mkocha H, Munoz B, Mabey D, Foster A, et al(2005) Risk factors for postsurgical trichiasis recurrence in a trachoma-endemic area. Invest Ophthalmol Vis Sci. 46:447-53.

[12] Mahande M, Tharaney M, Kirumbi E, Ngirawamungu E, Geneau R, et al. (2007) Uptake of trichiasis surgical services in Tanzania through two village based approaches. $\mathrm{Br} J$ Ophthalmol 91: 139-142.

[13] Roba AA, Wondimu A, Eshetu Z (2012) Effect of SAFE Intervention on Pattern of Barriers to Trichiasis Surgery, Ethiopia. $J$ Community Med Health Educ 2:162. doi:10.4172/2161-0711.1000162.

[14] Melese M, Alemayehu W, Friedlander E, Courtright P (2004) Indirect costs associated with accessing eye care services as a barrier to service use in Ethiopia. Trop Med Int Health 9: 426-431.

[15] Summary proceedings of the twelfth annual trachoma control program review of the Carter Center Atlanta, Georgia 2011; February, 22-24.

[16] The Ethiopian Journal of health development special issue on National blindness, low vision and trachoma survey in Ethiopia 2005 -2006 Ethiop.J.HealthDev.2007;21(3)204-205

[17] Summary proceedings of the twelfth annual trachoma control program review of the Carter Center Atlanta, Gorgia 2012; February, 27-29.

[18] Rabiu MM , Abiose A (2001) Magnitude of trachoma and barriers to uptake of lid surgery in a rural community of northern Nigeria. Ophthalmic Epidemiol 8(2-3):181-90

[19] Silvio P. Mariotti. Looking forward to Global elimination of trachoma by 2020 (Get 2020) Am.J.Trop med Hyg, 1988. 
[20] Baso Liben (woreda) - Wikipedia, the free encyclopedia. Available on http:/ /en.wikipedia.org/wiki/Baso Liben (woreda). Accessed on 19 may, 2013. 